\title{
Radionuclide Imaging of Infection: In Search of the Grail
}

\begin{abstract}
A
lthough there have been significant advances in the understanding of the pathogenesis of infection, it remains a major cause of patient morbidity and mortality throughout the world (1). The presence of infection may be suggested by signs and symptoms such as pain, fever, general malaise, and abnormal laboratory results, but imaging tests often are used to confirm the diagnosis. Radionuclide studies, in fact, have been used for detecting and localizing infection for nearly half a century. In addition to being both sensitive and specific, the ideal radiopharmaceutical for detecting infection must be nontoxic, affordable, widely available, and easily and rapidly prepared. The agent should
\end{abstract}

\section{See pages 790 and 823}

clear rapidly from the circulation both to facilitate early imaging and to minimize exposure of the patient to radiation. High-quality images also are important. Finally, it is desirable for the radiopharmaceutical to be able to monitor response to therapy. ${ }^{99 \mathrm{~m}} \mathrm{Tc}-$ methylene diphosphonate, ${ }^{67} \mathrm{Ga}$-citrate, ${ }^{18} \mathrm{~F}-\mathrm{FDG}$, and in vitro labeled leukocyte imaging all are useful; none, however, even approaches the ideal.

Leukocyte imaging, using cells labeled in vitro with ${ }^{111} \mathrm{In}$-oxine or ${ }^{99 m} \mathrm{Tc}$-exametazime, is currently the radionuclide gold standard for diag-

Received Jan. 26, 2009; revision accepted Jan. 29, 2009.

For correspondence or reprints contact: Christopher J. Palestro, Division of Nuclear Medicine and Molecular Imaging, Long Island Jewish Medical Center, 270-05 76th Ave., New Hyde Park, NY 11040.

E-mail: palestro@lij.edu

COPYRIGHT @ 2009 by the Society of Nuclear Medicine, Inc.

DOI: 10.2967/jnumed.108.058297 nosing most infections in the immunocompetent population. Technically demanding, the labeling process requires skilled personnel, is laborintensive, is not always available, and involves direct handling of blood products. Labeling a sufficient number of leukocytes to obtain images of diagnostic quality may not be possible in the severely leukopenic or very young patient. Image quality, especially when using ${ }^{111} \mathrm{In}$ as the radiolabel, is less than optimal. For musculoskeletal infection, the need to perform complementary marrow or bone imaging adds complexity and expense to the procedure and can be a burden to the patient who is elderly or debilitated, as often is the case (2).

In the typical clinical milieu, neutrophils-because they make up the largest number of circulating white cells-constitute the majority of leukocytes labeled. Hence, the procedure is most sensitive for identifying neutrophilmediated infections and thus is very useful for bacterial infection (2). Unfortunately, some noninfectious inflammatory conditions, acute rheumatoid and gouty arthritis, for example, also incite a neutrophilic response limiting, to some degree, the specificity of the test (3). Though valuable for detecting neutrophil-mediated infections, labeled leukocyte imaging is less useful, because of decreased sensitivity, for detecting those illnesses, such as Pneumocystis carinii pneumonia and tuberculosis, in which the predominant cellular response is not neutrophilic (4). The success of labeled leukocyte imaging is based not on its ability to detect infection per se but rather on its ability to detect neutrophil accumulation. The test is leukocyte-specific, not infection-specific.

Despite its value, the disadvantages of in vitro labeled leukocyte imaging are not insignificant, and numerous investigations have been devoted both to devising alternative methods of labeling white cells and to developing agents specific for infection. Efforts to overcome the limitations of in vitro labeling have focused on in vivo techniques that use whole antibodies, antibody fragments, peptides, and cytokines that bind to various receptors on white cells (5-11). Despite encouraging results reported in clinical trials, none of these agents ever has engendered widespread clinical use. None, moreover, is available in the United States, even on an investigational basis.

In the current issue of The Journal of Nuclear Medicine, Locke et al. (12) revisit the concept of in vivo leukocyte labeling, albeit with a somewhat different and provocative approach: using a positron, rather than a single-photon, emitter as the radiolabel. PET has important advantages over conventional $\gamma$-camera imaging using single-photon emitters. It intrinsically is a highresolution tomographic technique that enables precise localization of abnormalities. Semiquantitative analysis, readily available with PET but less feasible with conventional $\gamma$-camera imaging, perhaps could be useful for differentiating infectious from noninfectious conditions or for monitoring response to therapy. Indeed, ${ }^{18} \mathrm{~F}-\mathrm{FDG}$ PET already is assuming increasing importance in the diagnosis and localization of infection and inflammation $(13-16)$. Although it is exquisitely sensitive, ${ }^{18} \mathrm{~F}-\mathrm{FDG}$ is not specific and is concentrated by a variety of conditions, including malignant and benign neoplasms, fractures, and aseptic inflammation, as well as infection.

Initial attempts at developing a more specific PET tracer for infection imaging focused on labeling leukocytes in 
vitro with ${ }^{18}$ F-FDG $(17,18)$. Although the results obtained were satisfactory, there are considerable, likely insurmountable, limitations to this test. The labeling efficiency of ${ }^{18} \mathrm{~F}$-FDG leukocytes is more variable and significantly lower than that of ${ }^{111}$ In-oxine leukocytes $(2,17)$. The stability of the label is poor. In one animal investigation, more than $50 \%$ of the activity eluted from leukocytes within $90 \mathrm{~min}$ and about $80 \%$ eluted within 6 h (19). The short half-life $(110 \mathrm{~min})$ of ${ }^{18} \mathrm{~F}$ precludes delayed, next-day, imaging and makes the procedure practical only at those institutions that can perform labeling on-site. Thus, it is unlikely that infection imaging with ${ }^{18}$ F-FDG leukocytes ever will be clinically practical (2).

The work by Locke et al. (12) is a logical progression in the development of PET tracers for imaging infection: from ${ }^{18} \mathrm{~F}$-FDG to leukocytes labeled in vitro with ${ }^{18} \mathrm{~F}$-FDG and now to leukocytes labeled in vivo with ${ }^{64} \mathrm{Cu}$. The peptide used in their investigation, cinnamoyl-F-(D)L-F-(D) L-F (cFLFLF), is an antagonist to the neutrophil formyl peptide receptor with a high binding affinity (dissociation constant, $2 \mathrm{nM}$ ). Unlike some other peptides that have been investigated, cFLFLF does not exert any biologic effects on the leukocytes themselves (20). Because of high hydrophobicity, however, cFLFLF demonstrates relatively poor target-to-background ratios in imaging focal sites of infection (21). To improve the hydrophilicity of cFLFLF, the investigators coupled it with polyethylene glycol and chelated the pegylated peptide to ${ }^{64} \mathrm{Cu}$ with 2 , $2^{\prime}, 2^{\prime \prime}, 2^{\prime \prime \prime}$-(1,4,7,10-tetraazacyclododecane-1,4,7,10-tetray1) tetraacetic acid (DOTA).

The choice of ${ }^{64} \mathrm{Cu}$, which has a half-life of about $13 \mathrm{~h}$, as the radiolabel was prescient. The ideal interval between injection and imaging is governed by many factors including tracer clearance, intensity of the inflammatory process, and leukocyte kinetics. The peptide probably could have been labeled with the more ubiquitous positron emitter, ${ }^{18} \mathrm{~F}$. However, this would have required that imaging be per- formed within 5-6 h, rather than $18 \mathrm{~h}$ after injection, the optimum imaging time in this investigation. As shown in this investigation, the ability to perform delayed imaging is important.

In vitro, the radiolabeled peptide had a high binding affinity for human neutrophils and, unlike some other peptides, did not exert any biologic effects on the cells themselves. This finding is encouraging; monitoring cell viability over time would also have been useful.

Stability of the label is critical to the accuracy of the test. Sites of activity must reflect areas of leukocyte accumulation and not foci of nonspecific radioactivity. The fact that the stability of the peptide-neutrophil complex over time was not studied is unfortunate because the biodistribution data raise questions about the strength of the bond. Neutrophils are cleared from the circulation by the reticuloendothelial system; images obtained with labeled leukocytes, regardless of whether they are labeled in vitro or in vivo, are characterized by, at a minimum, activity in the liver, spleen, and bone marrow (2). In this investigation, however, splenic uptake was quite low, and bone or marrow activity was virtually nonexistent. The data in Table 3, which show high concentrations of radioactivity in the liver, kidneys, and small intestine, parallel more closely the biodistribution of ${ }^{64} \mathrm{Cu}$, or perhaps ${ }^{64} \mathrm{Cu}$ complexes, than that of neutrophils. Thus, one is left to ponder how much of the activity, at the time of imaging, actually was leukocyte-bound.

There was significantly more activity in the lungs of infected animals than in the lungs of controls. Histopathologic analysis confirmed the presence of large numbers of neutrophils in the lungs of infected rats and few, if any, in the lungs of controls. This finding implies, but does not prove, that the increased activity in the lungs of infected rats was due to infiltration of labeled neutrophils. The findings also could be explained on the basis of hyperemia and accumulation of free ${ }^{64} \mathrm{Cu}$ or ${ }^{64} \mathrm{Cu}$-peptide complexes in the lungs. The data would have been more convincing if the investigation also had included a group of neutropenic infected animals injected with radiolabeled peptide and compared with the controls and the infected animals.

Although the ability to perform in vivo labeled leukocyte PET would be a significant improvement over current technology, the test still is not specific for infection. Therefore, although some investigators pursue improved methodology for labeled leukocyte imaging, other investigators pursue infectionspecific tracers. One of the earliest attempts at developing an infectionspecific tracer involved the use of radiolabeled antibiotics. The radiolabeled antibiotic, theoretically, would be incorporated into and metabolized by bacteria and would make possible the accurate and specific localization of the site of infection. The most extensively investigated of these agents was the radiolabeled fluoroquinolone 99mTc-ciprofloxacin. Published results over several years, however, have been contradictory, and the original enthusiasm for this agent has waned (22).

More recent investigations have focused on radiolabeled antimicrobial peptides, which play a critical role in the biologic defense system of multicellular organisms against bacteria, fungi, and viruses. These peptides are produced by various cells, including phagocytes and endothelial and epithelial cells, and bind to the bacterial cell membrane. Expression of these peptides may be constant or induced on contact with microbial organisms; the peptides also may be transported to sites of infection by leukocytes (23).

In this issue of The Journal of Nuclear Medicine, Liberatore et al. (24) report on microbial targeting with a ${ }^{99 \mathrm{~m}}$ Tc-labeled antimicrobial peptide, recombinant human $\beta$-defensin-3, that exerts bactericidal effects on grampositive and gram-negative bacteria, as well as on some yeasts. The investigators demonstrated that by $3 \mathrm{~h}$ after injection, tracer uptake in a Staphylococcus aureus infection model was significantly higher than uptake in sterile inflammation, lending credence to the concept of a "specific" infection imaging agent. 
An agent that is specific for infection is desirable; high specificity at the expense of sensitivity is not. There are at least 3 factors that potentially could affect the sensitivity of radiolabeled antimicrobial peptides: the causative organism, the number of bacteria available for binding, and the host response to infection. Liberatore et al. (24) selected $S$. aureus rather than Escherichia coli for the in vivo part of the investigation because, in vitro, ${ }^{99 m}$ Tc-recombinant human $\beta$-defensin-3 demonstrated greater inhibition of bacterial growth with $S$. aureus. Would the differences in tracer uptake between infection and inflammation have been as striking in E. coli infection? It is unfortunate that the investigators did not study both organisms.

The sensitivity of antimicrobial peptides also may be related to the number of bacteria available for binding. Ahktar et al. (25) investigated the antimicrobial peptide ${ }^{99 \mathrm{~m} T c-u b i q u i c i d i n}$ (29-41) and found that uptake was related to the number of viable bacteria present. They also observed that uptake decreased after treatment with ciprofloxacin. Sarda-Mantel et al. (26) evaluated an animal model of prosthetic joint infection with the same agent. They observed that although all 6 infected devices were positive on day 9 , only 4 of the 6 were positive on day 20. These investigators speculated that decreased sensitivity over time may have been related to the effects of the protective biofilm or glycocalix secreted by the bacteria. Would the results of Liberatore et al. (24) have been different had they injected greater or lesser quantities of bacteria into the animals or had they increased or decreased the interval between inoculation and tracer injection?

Host response to infection could potentially affect the sensitivity of radiolabeled antimicrobial peptides (27). How sensitive will these agents be in the immunosuppressed or immunocompromised host? This is an issue that has yet to be addressed.

And so the quest for the grail continues. Although Locke et al. (12) and
Liberatore et al. (24) approach the radionuclide diagnosis of infection from rather different perspectives, their investigations should be viewed as complementary rather than competitive. None of the currently available radiotracers work equally well in all situations; labeled leukocytes perform better in certain circumstances, gallium in others, and ${ }^{18}$ F-FDG in still others. Similarly, in vivo PET labeled leukocyte imaging might be useful in some circumstances, whereas antimicrobials could be useful in others. Ultimately, the radionuclide grail of infection imaging likely will contain not a single "ideal" tracer but several from which we can choose.

Christopher J. Palestro North Shore Long Island Jewish Health System

New Hyde Park, New York

\section{REFERENCES}

1. Kok M, Pechère JC. Nature and pathogenicity of micro-organisms. In: Cohen, J, Powderly, WG, eds. Infectious Diseases. St. Louis, MO: Mosby; 2004:3-29.

2. Palestro CJ, Love C, Bhargava KK. Labeled leukocyte imaging: current status and future directions. Q J Nucl Med Mol Imaging. 2009;53: 105-123.

3. Palestro CJ, Love C, Miller TT. Imaging of musculoskeletal infections. Best Pract Res Clin Rheumatol. 2006;20:1197-1218.

4. Fineman DS, Palestro CJ, Kim CK, et al. Detection of abnormalities in febrile AIDS patients with In-111-labeled leukocyte and Ga-67 scintigraphy. Radiology. 1989;170:677-680.

5. Weiner RE, Thakur ML. Imaging infection/inflammations. Q J Nucl Med. 1999;43:2-8.

6. Becker W, Goldenberg DM, Wolf F. The use of monoclonal antibodies and antibody fragments in the imaging of infectious lesions. Semin Nucl Med. 1994;24:142-153.

7. Thakur ML, Marcus CS, Henneman P, et al. Imaging inflammatory diseases with neutrophilspecific technetium-99m-labeled monoclonal antibody anti-SSEA-1. J Nucl Med. 1996;37:17891795.

8. Palestro CJ, Kipper SL, Weiland FL, Love C, Tomas MB. Osteomyelitis: diagnosis with ${ }^{99 \mathrm{~m} T c}$ labeled antigranulocyte antibodies compared with diagnosis with ${ }^{111}$ In-labeled leukocytes-initial experience. Radiology. 2002;223:758-764.

9. Becker W, Blair J, Behr T, et al. Detection of soft tissue infections and osteomyelitis using a technetium-99m-labeled anti-granulocyte monoclonal antibody fragment. J Nucl Med. 1994;35: 1436-1443.
10. Palestro CJ, Weiland FL, Seabold JE, et al. Localizing infection with a technetium labeled peptide: initial results. Nucl Med Commun. 2001; 22:695-701.

11. Bleeker-Rovers CP, Rennen HJ, Boerman OC, et al. ${ }^{99 \mathrm{~m} T c-l a b e l e d ~ i n t e r l e u k i n ~} 8$ for the scintigraphic detection of infection and inflammation: first clinical evaluation. J Nucl Med. 2007;48:337343.

12. Locke LW, Chordia MD, Zhang Y, et al. A novel neutrophil-specific PET imaging agent: cFLFLFPEG- ${ }^{64} \mathrm{Cu}$. J Nucl Med. 2009;50:790-797.

13. Zhuang H, Alavi A. 18-fluorodeoxyglucose positron emission tomographic imaging in the detection and monitoring of infection and inflammation. Semin Nucl Med. 2002;32:47-59.

14. Love C, Tomas MB, Tronco GG, Palestro CJ. Imaging infection and inflammation with ${ }^{18} \mathrm{~F}$-FDGPET. Radiographics. 2005;25:1357-1368.

15. Strobel K, Stumpe KD. PET/CT in musculoskeletal infection. Semin Musculoskelet Radiol. 2007; 11:353-364.

16. Bleeker-Rovers CP, Vos FJ, Corstens FH, Oyen WJ. Imaging of infectious diseases using $\left[{ }^{18} \mathrm{~F}\right]$ fluorodeoxyglucose PET. $Q \mathrm{~J} \mathrm{Nucl} \mathrm{Med} \mathrm{Mol}$ Imaging. 2008;52:17-29.

17. Rini JN, Bhargava KK, Tronco GG, et al. ${ }^{18}$ F-FDG in vitro labeled autologous human leukocytes versus ${ }^{111}$ In-labeled leukocytes for the detection of infection. Radiology. 2006;238:978-987.

18. Dumarey N, Egrise D, Blocklet D, et al. Imaging infection with ${ }^{18}$ F-FDG-labeled leukocyte PET/ CT: initial experience in 21 patients. $\mathrm{J}$ Nucl Med. 2006;47:625-632.

19. Pellegrino D, Bonab AA, Dragotakes SC, Pitman JT, Mariani G, Carter EA. Inflammation and infection: imaging properties of ${ }^{18}$ F-FDG-labeled white blood cells versus ${ }^{18}$ F-FDG. J Nucl Med. 2005;46:1522-1530.

20. Derian CK, Solomon HF, Higgins JD, et al. Selective inhibition of $\mathrm{N}$-formylpeptide-induced neutrophil activation by carbamate-modified peptide analogues. Biochemistry. 1996;35:1265-1269.

21. Babich JW, Dong Q, Graham W, et al. A novel high affinity chemotactic peptide antagonist for infection imaging [abstract]. J Nucl Med. 1997; 38(suppl):268P.

22. Benitez A, Roca M, Martin-Comin J. Labeling of antibiotics for infection diagnosis. $Q \mathrm{~J} \mathrm{Nucl} \mathrm{Med}$ Mol Imaging. 2006;50:147-152.

23. Lupetti A, Pauwels EKJ, Nibbering PH, Welling MM. ${ }^{99 \mathrm{~m}} \mathrm{Tc}$-antimicrobial peptides: promising candidates for infection imaging. Q J Nucl Med. 2003; 47:238-245.

24. Liberatore M, Pala A, Scaccianoce S, et al. Microbial targeting of ${ }^{99 \mathrm{~m}} \mathrm{Tc}$-labeled recombinant human $\beta$-defensin-3 in an animal model of infection: a feasibility pilot study. J Nucl Med. 2009;50:823-826.

25. Akhtar MS, Khan ME, Khan B, et al. An imaging analysis of ${ }^{99 m}$ Tc-UBI (29-41) uptake in S. aureus infected thighs of rabbits on ciprofloxacin treatment. Eur J Nucl Med Mol Imaging. 2008;35: 1056-1064.

26. Sarda-Mantel L, Saleh-Mghir A, Welling MM, et al. Evaluation of $99 \mathrm{~m}$ Tc-UBI 29-41 scintigraphy for specific detection of experimental Staphylococcus aureus prosthetic joint infections. Eur J Nucl Med Mol Imaging. 2007;34:1302-1309.

27. Signore A, D'Alessandria C, Lazzeri E, Dierckx R. Can we produce an image of bacteria with radiopharmaceuticals? Eur J Nucl Med Mol Imaging. 2008;35:1051-1055. 